\title{
Obstrucción intestinal debido a hernia estrangulada en el espacio prevesical: presentación de un caso y revisión de la literatura
}

\author{
Beatriz Laiz Díez, Juan González González¹, Jaime Ruiz Tovar ${ }^{1}$ \\ Matías Cea Soriano ${ }^{2}$ y Manuel Durán Poveda ${ }^{2}$
}

Intestinal obstruction caused by strangulated hernia in the prevesical space: One case and revision of the literature

Introduction: Prevesical space is an infrequent location of hernias. Prevesical hernia is a rare cause of bowel obstruction. We present a case operated at our institution and review the available evidence in literature. Case report: A 67-years-old mail, without any relevant medical history, came to the Emergency Department complaining of suprapubic pain during the last $24 \mathrm{~h}$. Physical examination revealed abdominal distension and suprapubic pain. A CT scan revealed small bowel dilation with a gauge change in the pelvis. Exploring laparotomy was performed, observing a hernia in the prevesical space and $25 \mathrm{~cm}$ of ileal loops incarcerated with ischemic aspect. A small bowel resection and latero-lateral manual running suture anastomosis was performed. Histological findings were not relevant. The patient presented an uneventful postoperative course and was discharged the 5th day after surgery. Conclusions: Hernias in the prevesical space are infrequent causes of abdominal pain and bowel obstruction. They are located between lateral umbilical ligaments and bladder fundus, where a ring is located. They must be suspected in cases of inguinal or suprapubic pain without palpable lump in the lower abdominal quadrants. Diagnosis can be only radiologically achieved, by CT scan. Surgical treatment is mandatory, often requiring bowel resection.

Key words: Prevesical space; Hernia; Intestinal obstruction.

\section{Resumen}

Introducción: El espacio prevesical es una localización infrecuente de hernia. La hernia prevesical es causa infrecuente de obstrucción intestinal. Presentamos el caso de un paciente intervenido en nuestro centro y realizamos una revisión de la literatura. Caso clínico: Varón de 67 años sin antecedentes médicoquirúrgicos de interés que acude a Urgencias por dolor suprapúbico de 24 h de evolución. A la exploración destacan distensión abdominal y dolor en la localización previamente descrita. Se realiza estudio analítico completo y TC abdominal, en el que se objetiva dilatación de asas del intestino delgado con cambio de calibre a nivel pélvico. Se decide realizar laparotomía exploradora: herniación de $25 \mathrm{~cm}$ de íleon de aspecto isquémico en el espacio prevesical. Se realiza resección intestinal y anastomosis enteroentérica L-L manual monoplano. El estudio anatomopatológico de la pieza quirúrgica no revela otros datos de interés. El paciente presenta buena evolución postoperatoria con tolerancia oral progresiva y es dado de alta al quinto día de la intervención. Conclusiones: Las hernias del espacio prevesical son causa infrecuente de dolor abdominal y de obstrucción intestinal. Se localizan entre los ligamentos umbilicales laterales y el fundus de la vejiga, donde se forma un anillo peritoneal. Deben sospecharse ante dolor inguinal o suprapúbico sin tumoración palpable en el piso abdominal inferior. El diagnóstico es radiológico, mediante TC. El tratamiento es quirúrgico y ocasionalmente puede precisar resección intestinal.

Palabras clave: Espacio prevesical; Hernia; Obstrucción intestinal.
Hospital Universitario Rey Juan Carlos I, Madrid, España. ${ }^{2}$ Hospital Universitario Puerta de Hierro, Majadahonda, Madrid, España.

Recibido el 21 de agosto de 2016, aceptado el 10 de enero de 2017.

Correspondencia a: Beatriz Laiz Díez bealaiz@hotmail.com 


\section{Introducción}

La hernia en el espacio prevesical es una entidad extremadamente infrecuente, existiendo pocos casos descritos en la literatura. Consiste en que el saco herniario se introduce en la reflexión peritoneal que cubre el espacio prevesical o espacio de Retzius. Clínicamente se manifiesta como una hernia interna, ocasionando un cuadro de obstrucción y requiere intervención quirúrgica urgente.

\section{Caso clínico}

Presentamos el caso de un varón de 72 años con hipertensión arterial en tratamiento médico como único antecedente personal de interés, quien acudió al servicio de Urgencias por cuadro de dolor abdominal, vómitos y ausencia de deposición de 4 días de evolución, asociado a pico febril de $38^{\circ} \mathrm{C}$.

En la exploración física destacó un abdomen distendido, doloroso de forma difusa, más llamativo en hipogastrio, sin signos de irritación peritoneal. Los ruidos hidroaéreos estaban ausentes. No se palparon masas, visceromegalias ni hernias. El tacto rectal se mostró sin hallazgos.

Una radiografía de abdomen objetivó dilatación de asas del intestino delgado y niveles hidroaéreos. No se observó neumoperitoneo. En la analítica de sangre destacaba una creatinina de $1,8 \mathrm{mg} / \mathrm{dl}$, una leucocitosis de $22.000 / \mathrm{mm}^{3}$ con $88,4 \%$ de neutrófilos y una actividad de protrombina del $65 \%$ con un INR de 1,36 .

Se procedió a la colocación de una sonda nasogástrica obteniendo débito fecaloideo a través de la misma en moderada cuantía. Se mantuvo en dieta absoluta con pauta de sueroterapia y se solicitó TC abdominal urgente, donde se apreció dilatación de asas del intestino delgado hasta una imagen en asa cerrada en yeyuno localizada en hipogastrio a partir de la cual las asas del intestino delgado recuperaban un calibre normal sin evidencia de causa obstructiva. Se objetivó neumatosis en las paredes del asa cerrada así como importante afectación de la grasa mesentérica circundante (Figuras 1 y 2). Ante la sospecha de una brida o una hernia interna que condicionaran el cuadro clínico y ante la situación de sepsis del paciente se decidió laparotomía exploradora.

Se realizó abordaje mediante laparotomía media suprainfraumbilical visualizándose hernia interna en el espacio prevesical de $15 \mathrm{~cm}$ de yeyuno en un fondo de saco revestido de peritoneo. Se observó necrosis de asa intestinal (Figura 3) y líquido libre de sufrimiento intestinal. Se realizó resección intestinal y anastomosis latero-lateral. Se efectuó una puesta a plano del fondo de saco desperitonizándolo. El postoperatorio cursó sin incidencias, con tolerancia progresiva a alimentación vía oral, siendo dado de alta al quinto día postoperatorio.

El seguimiento posterior en consulta fue satisfactorio, permaneciendo el paciente asintomático.

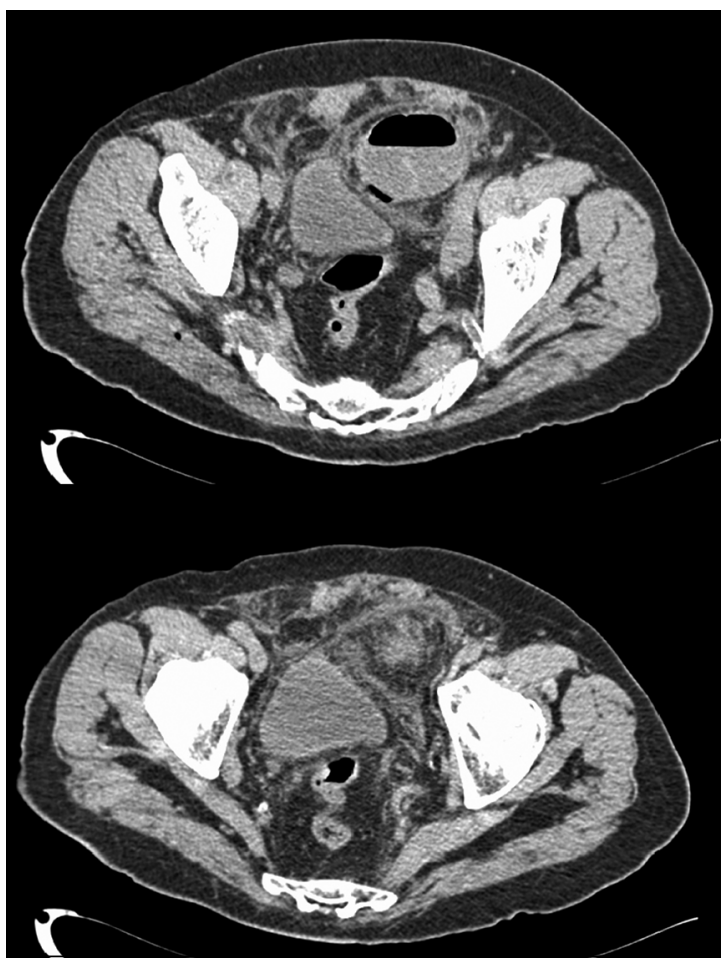

Figura 1 y 2. Cortes de TC abdominopélvico donde se objetiva atrapamiento de un asa del intestino delgado en el espacio prevesical.

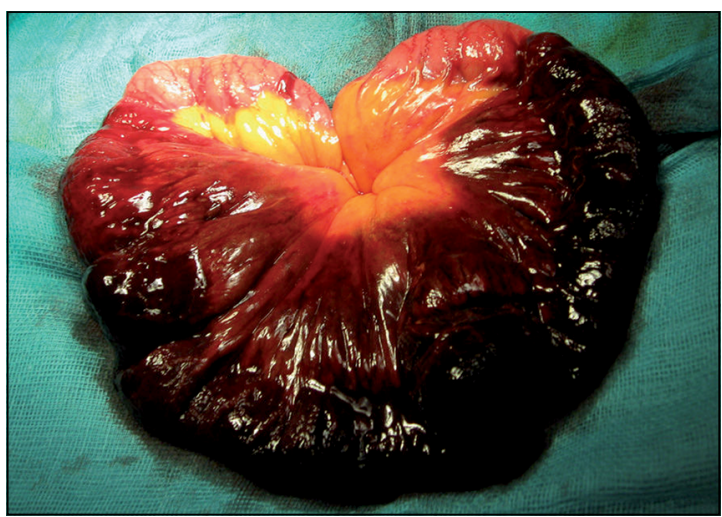

Figura 3. Segmento de yeyuno con isquemia intestinal. 


\section{Discusión}

La hernia en el espacio prevesical o espacio de Retzius se localiza, como su propio nombre indica, en esta región, más concretamente en la fosa supravesical. Esta es un área triangular relacionada lateralmente y por encima con los pliegues umbilicales laterales que cubren las arterias hipogástricas obliteradas y, por debajo, adherida a una reflexión peritoneal que se extiende desde la pared abdominal anterior hasta el fundus vesical ${ }^{1}$.

Las hernias asentadas en esta localización se forman como resultado de un fallo en la integridad de la aponeurosis del músculo transverso y la fascia transversalis y normalmente protuyen hacia la pared abdominal como una hernia inguinal directa (hernia supravesical externa). Sin embargo, raramente, protuyen hacia el abdomen (hernia supravesical interna) $)^{1,2-4}$.

Muchos factores influyen en la aparición de este tipo de hernias. En primer lugar, un aumento de la prominencia de los pliegues umbilicales (en ocasiones producidas por anomalías en la regresión de estructuras fetales), que dan lugar a la formación de bolsillos peritoneales, por donde se pueden herniar estructuras a través de áreas debilitadas ${ }^{1}$. La atrofia de la grasa subperitoneal es un factor adicional que colabora en la formación de invaginaciones del peritoneo, al igual que la existencia de inflamación por debajo del peritoneo, que dan lugar a la formación de cicatrices por donde se producen las hernias. Los defectos congénitos del tejido conectivo o adquiridos, como en el caso de los fumadores, pueden dar lugar a la aparición de estas hernias asociadas en más de la mitad de los casos a hernias inguinales'. El aumento de la presión intraabdominal es un factor que colabora en la formación de este tipo de hernias.

La hernia supravesical interna no se manifiesta como tumoración palpable en superficie debido a que la presencia de la sínfisis del pubis evita la exteriorización de la misma. Los principales síntomas están en relación con el cuadro de obstrucción intestinal que puede producir la hernia incarcerada o estrangulada, en ausencia de cirugías abdominales previas y ausencia de tumoración inguinal palpable ${ }^{3,5}$.

El TC abdominopélvico es la prueba con mayor rentabilidad diagnóstica en la hernia prevesical, siendo esta próxima al $90 \%$, evidenciando asas intestinales localizadas anteriores a la vejiga, en el espacio prevesical. La cistoscopia igualmente puede evidenciar una prominencia en la pared anterior de la vejiga. La RM también puede ser de utilidad $^{3,4,6}$.

El tratamiento inicial puede ser conservador mediante colocación de sonda nasogástrica y reposición hidroelectrolítica, pero ante situaciones de sospecha clínica, analítica o mediante pruebas de imagen, de isquemia o perforación intestinal, será necesaria la cirugía urgente. En el caso de la hernia prevesical, dado que el cuello del saco herniario es rígido y estrecho, es frecuente la estrangulación del asa intestinal, por lo que habitualmente precisa tratamiento quirúrgico, que consistirá en la reducción de la hernia y sutura del orificio ${ }^{3,4}$. La laparoscopia es aceptada como método diagnóstico y terapéuti$\mathrm{co}^{3,4,7}$. El pronóstico depende fundamentalmente de la viabilidad intestinal en el momento de la cirugía.

\section{Responsabilidades éticas}

Protección de personas y animales. Los autores declaran que para esta investigación no se han realizado experimentos en seres humanos ni en animales.

Confidencialidad de los datos. Los autores declaran que han seguido los protocolos de su centro de trabajo sobre la publicación de datos de pacientes.

Derecho a la privacidad y consentimiento informado. Los autores han obtenido el consentimiento informado de los pacientes y/o sujetos referidos en el artículo. Este documento obra en poder del autor de correspondencia.

\section{Financiación}

El presente trabajo no posee fuente de financiación.

\section{Conflicto de intereses}

Los autores declaran no tener ningún conflicto de intereses. 


\section{CASO CLÍNICO}

\section{Bibliografía}

1. Sozen I, Nobel J. Inguinal mass due to an external supravesical hernia and acute abdomen due to an internal supravesical hernia: A case report and review of the literature. Hernia. 2004;8:389-92.

2. Skandalakis JE, Gray SW, Burns WB, Sangmalee U, Sorg JL. Internal and external supravesical hernia. Am Surg. 1976;42:142-6.
3. Bouassida M, Sassi S, Touinsi H, Kallel $\mathrm{H}$, Mighri MM, Chebbi F, et al. Internal supravesical hernia: A rare cause of intestinal obstruction, report of two cases. Pan Afr Med J. 2012;11:17.

4. Cissé M, Konaté I, Ka O, Dieng M, Dia A, Touré CT. Internal supravesical hernia as a rare cause of intestinal obstruction: A case report. J Med Case Reports. 2009;3:9333.

5. Sánchez Fuentes PA, García Zamora C, Pĩnero Madrona A, Parrila Paricio P.
Obstrucción intestinal causada por hernia supravesical interna. Cir Esp. 2015;93.

6. Saravanan B, Paramu MKA, Ranganathan L. Supravesical hernia. A rare cause of intestinal obstruction. Int J Surg. 2008;6:471-2.

7. Kawaguchi T, Itoh T, Yoshii K, Otsuji E. Laparoscopic repair of a bilateral internal inguinal hernia with supravesical hernia. A case report. Int J Surg Case Rep. 2015;14:108-11. 